\title{
RECUENTO DE ACTINOMYCES EN SALIVA COMO PREDICTORES MICROBIOLÓGICO DE CARIES
}

\author{
COUNT OF ACTINOMYCES IN SALIVA AS MICROBIOLOGICAL PREDICTORS OF CARIES
}

\author{
Susana Gutiérrez de Annan * \\ Laura Benito de Cárdenas **
}

\begin{abstract}
RESUMEN
Introduction: La caries dental es definida como un proceso infeccioso, multifactorial y crónico. Actualmente se realiza el recuento en saliva del grupo Streptococcus mutans, Lactobacillus y Candida. El género Actinomyces se considera agente etiológico de caries, pero no existen pruebas que hayan evaluado el nivel de este microorganismo en saliva. El objetivo de este trabajo es determinar la correlación entre los niveles de Actinomyces en saliva y los indicadores clínicos de riesgo y actividad cariogénica para utilizar el recuento de estos microorganismos como predictor de riesgo de caries. Métodos: 33 estudiantes entre 19 y 24 años, de ambos sexos, fueron incluidos en este estudio. Los mismos se clasificaron en 3 grupos: sanos, con riesgo cariogénico y con actividad cariogénica según los siguientes indicadores clínicos: Índice O’Leary, No de caries amelodentinarias abiertas, frecuencia de cepillado y momentos de azúcar. Muestras de saliva fueron sembradas en Agar Brucella con hemina, vitamina K y 5\% de sangre. Se incubó en anaerobiosis por 7 días y se realizó el recuento de Actinomyces y microbiota acompañante. Resultados: Hubo diferencias estadísticamente significativas en el recuento de la microbiota total y los niveles en saliva de Actinomyces, entre los estudiantes sanos con los que poseen riesgo y aquellos con actividad cariogénica. Conclusión: El presente estudio conduce a proponer la utilización del recuento de Actinomyces como predictores de riesgo de caries conjuntamente con el recuento del grupo Streptococcus mutans, Lactobacillus y Candida.
\end{abstract}

DESCRITORES: Actinomyces $\bullet$ Saliva $\bullet$ Caries dental

\begin{abstract}
Introduction: Dental caries is defined as an infectious, multifactorial and chronic process. At the present time there is carried out the recount in saliva of the group Streptococcus mutans, Lactobacillus and Candida. The genus Actinomyces is considered an etiological agent of caries, but there are not tests that could evaluate the level of this microorganism in saliva. The aim of this work is to determine the correlation among the levels of Actinomyces in saliva and the clinical indicators of risk and cariogenic activity to use the recount of these microorganisms as predictors of caries risk. Methods: 33 students between 19 and 24 years, of both sexes, were included in this study. The same ones were classified in 3 groups: healthy, with cariogenic risk and with cariogenic activity according to the following clinical indicators: Index O'Leary, No of amelodentinal caries opened, brushing frequency and moments of sugar. Samples of saliva were inoculated in Brucella Agar with hemina, vitamin K and 5\% of blood. They were incubated in anaerobic jars during 7 days and the recount of Actinomyces and accompanying microbiota was made. Results: There were differences statistically significant in the recount of the total microbiota and the levels of Actinomyces in saliva, among the group of healthy students with those that had risk and the ones with cariogenic activity. Conclusion: The present study aims to propose the use of the recount of Actinomyces as predictors of caries risk jointly with the recount of the group Streptococcus mutans, Lactobacillus and Candida.
\end{abstract}

DESCRIPTORS: Actinomyces $\bullet$ Saliva $\bullet$ Dental caries

\footnotetext{
* Jefe de Trabajos Prácticos. Cátedra de Microbiología y Parasitología. Facultad de Odontología. Universidad Nacional de Tucumán. E-Mail: sannan@arnet. com.ar o susana.gutierrez@odontologia.unt.edu.ar

** Profesora Titular. Cátedra de Microbiología y Parasitología. Facultad de Odontología. Universidad Nacional de Tucumán. E-Mail: gjcardenas@infovia.com.ar
} 
Gutiérrez de Annan S, Benito de Cárdenas L. Recuento de actinomyces en saliva como predictores microbiológico de caries Revista de Odontologia da Universidade Cidade de São Paulo 2009 jan-abr; 21(1): 6-13

\section{INTRODUCCIÓN}

La caries dental ha sido objeto de estudio desde hace muchísimo tiempo, ya que es una de las enfermedades más comunes en el hombre. Esta enfermedad infecciosa, comúnmente crónica y multifactorial, se establece en la cavidad bucal mucho tiempo antes de producirse las manifestaciones clínicas de la misma, por lo que su predicción es importante antes de que se hayan desarrollado las lesiones visibles de caries. (Axelsson et al. ${ }^{2}$, 1991) (Elderton ${ }^{11}$, 1992) $\left(\right.$ Fejerskov $^{13}$, 1997) (Loyo Molina et $\left.a l^{20}, 1999\right)$. Cuando se realiza un diagnóstico predictivo, es importante obtener información acerca de los factores etiológicos de la caries bajo el concepto multifactorial. (Almagro-Nievas et al $\left.l^{1}, 2001\right)$ (Van Houte ${ }^{24}, 1993$ )

Los factores etiológicos principales involucrados en el establecimiento de la enfermedad son: un huésped susceptible, microorganismos cariogénicos, una dieta rica en sacarosa, la saliva y el tiempo en que interactúan estos factores.

La saliva es importante por su acción de autolimpieza y su alta capacidad buffer. Mantiene la integridad de los tejidos duros y posee acción antibacteriana. El flujo salival adecuado es también importante para determinar el riesgo de caries. (Loyo Molina et al20, 1999).

Para evaluar el papel cariogénico de la dieta, deberán tenerse en cuenta: la composición, la cantidad y la frecuencia del consumo de hidratos de carbono.

El biofilm de la placa dental como factor de riesgo es muy variable, pues depende de la cantidad de placa presente en la pieza dentaria y de la composición de la microbiota. La virulencia o cariogenicidad de los microorganismos bucales depende de las características metabólicas de los mismos. (Baca García y Liébana Ureña ${ }^{3}$, 1995)

Otros numerosos factores biológicos deben ser tenidos en cuenta ya que pueden favorecer la instalación de la enfermedad, conocidos como factores secundarios. Entre ellos, tenemos: la educación, la posición social y la ocupación del paciente. Otro punto a tener en cuenta es el conocimiento, la actitud y la conducta frente a esta enfermedad. Por otro lado, debemos informarnos sobre el padecimiento de enfermedades e ingestión de medicamentos que disminuyen el flujo salival. (Bellini $e t$ al 1981) (Fejerskov ${ }^{13}$, 1997) (Hausen ${ }^{16}$, 1997) (Ismail ${ }^{19,}$ 1997) (Tenovuo ${ }^{22}$, 1997). Estos datos son considerados de gran valor para determinar el riesgo de caries y están fuertemente asociados con los hábitos dietéticos. (Loyo Molina et $a l^{20}$, 1999) (Balda Zavarce et al ${ }^{4}$, 1999) (Fejer- skov $^{12}$, 2004) (van Palenstein Helderman et al ${ }^{5}$, 2006).

Los factores etiológicos principales y secundarios actualmente planteados intervienen conjuntamente y desencadenan esta enfermedad multifactorial. (Fejerskov ${ }^{13}$, 1997)

Numerosos estudios, a nivel mundial, incluyen el recuento en saliva del grupo Streptococcus mutans y Lactobacillus como una herramienta para predecir la caries dental. Nuevos estudios incluyen también el recuento de levaduras para tal fin. (Van Houte ${ }^{24}$, 1993) (Benito de Cárdenas et $a l^{9}$, 1994) (Conteli et $\left.a l^{10}, 2002\right)$. (Gutiérrez Acero $\left.e a l^{15}, 2006\right)$ (Beighton $\left.{ }^{6}, 2005\right)$ (Thenisch et $a l^{23}$, 2006)

Si bien Actinomyces se considera agente etiológico de caries, especialmente de caries de cemento, no existen estudios hasta al presente que indiquen una prueba que permita evaluar el nivel de este microorganismo en saliva como método preventivo de caries.

El objetivo del presente trabajo es determinar la posible correlación entre los niveles de Actinomyces en saliva y los indicadores clínicos odontológicos de riesgo y actividad cariogénica para que el recuento de estos microorganismos pueda ser considerado como predictor de riesgo de caries dental.

\section{MÉTODOS}

Población estudiada: se seleccionaron 33 estudiantes, de ambos sexos, con edades comprendidas entre 19 y 24 años. Los mismos cursaban $2^{\circ}$ año de la Carrera de Odontología de la Facultad de Odontología de la Universidad Nacional de Tucumán y que acudieron espontáneamente para este estudio.

Examen clínico: se determinó Índice de placa (IP) O'Leary, No de Caries Amelodentinarias Abiertas (CADA), Frecuencia de Cepillado (FC) y Momentos de Azúcar (MA). Según estos indicadores clínicos, los estudiantes fueron clasificados en 3 grupos: sanos, con riesgo cariogénico y con actividad cariogénica. En la Tabla 1, se observan las medias aritméticas $(\mathrm{x})$ y la desviación estándar (SD) de cada grupo.

Examen microbiológico: A cada estudiante se le solicitó, antes de realizar la higiene oral, una muestra de saliva sin estimular, en ayunas, que fue recogida en frasco estéril y procesada dentro de las 2 horas en el laboratorio. Se tuvo en cuenta que el alumno no estaba medicado con antibióticos.

Las muestras fueron dispersadas en agitador Vortex durante 1 minuto. Se sembró, por duplicado, $5 \mu \mathrm{l}$ en 
Gutiérrez de Annan S, Benito de Cárdenas L. Recuento de actinomyces en saliva como predictores microbiológico de caries Revista de Odontologia da Universidade Cidade de São Paulo 2009 jan-abr; 21(1): 6-13

Tabla 1: Características de la población estudiada

\begin{tabular}{|c|c|c|c|c|c|c|}
\hline GRUPOS & $\mathrm{n}$ & Edad (años) & $\begin{array}{c}\text { Índice de Placa } \\
(\%)\end{array}$ & $\begin{array}{c}\text { Caries } \\
\text { Amelodentinarias } \\
\text { Abiertas }\end{array}$ & $\begin{array}{l}\text { Frecuencia de } \\
\text { cepillado }\end{array}$ & $\begin{array}{c}\text { Momentos de } \\
\text { azúcar }\end{array}$ \\
\hline & & $\mathrm{x} \pm \mathrm{SD}$ & $\mathrm{x} \pm \mathrm{SD}$ & $\mathrm{x} \pm \mathrm{SD}$ & $\mathrm{x} \pm \mathrm{SD}$ & $\mathrm{x} \pm \mathrm{SD}$ \\
\hline Sano & 10 & $20 \pm 2$ & $19 \pm 7$ & $0 \pm 0$ & $3 \pm 1$ & $3 \pm 0$ \\
\hline Con Riesgo cariogénico & 10 & $21 \pm 2$ & $35 \pm 11$ & $0 \pm 0$ & $3 \pm 1$ & $4 \pm 1$ \\
\hline Con Actividad cariogénica & 13 & $20 \pm 1$ & $44 \pm 16$ & $3 \pm 1$ & $3 \pm 1$ & $5 \pm 1$ \\
\hline
\end{tabular}

cajas de Petri que contenían Agar Brucella con el agregado de hemina, vitamina K y 5\% de sangre. Se incubó a $37^{\circ} \mathrm{C}$ en jarra para anaerobios durante 7 días para recuperar los microorganismos de crecimiento lento.

Se realizó el recuento de las colonias desarrolladas y se procedió a la identificación bioquímica de especies según el Manual de Bergey 9o Ed. (Garrity et al ${ }^{14}$, 2001) (Johnson et al ${ }^{17}$, 1990) (Kandler y Weiss ${ }^{18}$, 1986).

Para analizar los recuentos de Actinomyces y microbiota total entre los 3 grupos de estudiantes (sanos, con riesgo cariogénico y con actividad cariogénica) se aplicó la Prueba de Kruskal-Wallis y Comparación Múltiple (Prueba de Dunn). Para determinar la asociación de es- tos microorganismos con los parámetros clínicos se utilizó el Coeficiente de Correlación de Spearman.

\section{RESULTADOS}

1) Población en estudio. En la Tabla 1 se describen las características de la población estudiada, indicando las medias aritméticas y la desviación estándar en cada grupo.

2) Recuento en saliva de diferentes tipos microbianos: El recuento de bacilos gram-positivos y gramnegativos, como así también los cocos gram-positivos y gram-negativos aumentan significativamente $(\mathrm{p}<0,05)$ en los estudiantes con riesgo y actividad cariogénica. Los

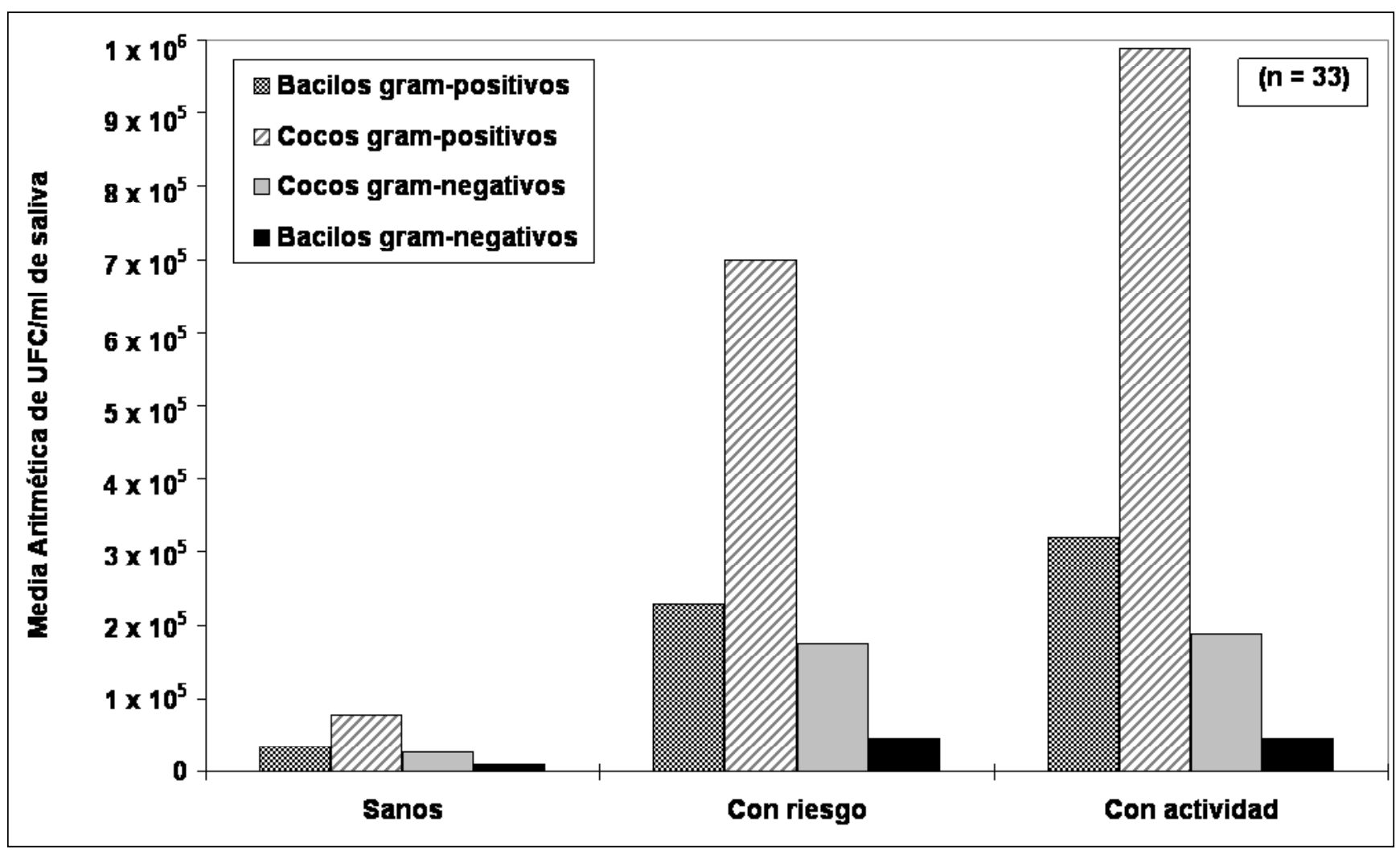

Figura 1: Recuento en Saliva de la Microbiota Total 
Gutiérrez de Annan S, Benito de Cárdenas L. Recuento de actinomyces en saliva como predictores microbiológico de caries Revista de Odontologia da Universidade Cidade de São Paulo 2009 jan-abr; 21(1): 6-13

Tabla 2: Recuento en saliva de los diferentes tipos microbianos (UFC/ml de saliva)

\begin{tabular}{cccc}
\hline \hline $\begin{array}{c}\text { Grupos } \\
\text { Microbiota aislada }\end{array}$ & $\begin{array}{c}\text { Sanos } \\
\mathrm{x} \pm \mathrm{SD}\end{array}$ & $\begin{array}{c}\text { Con Riesgo Cariogénico } \\
\mathrm{x} \pm \text { SD }\end{array}$ & $\begin{array}{c}\text { Con Actividad Cariogénica } \\
\mathrm{x} \pm \mathrm{SD}\end{array}$ \\
\hline Bacilos gram-positivos & $3,4 \times 104 \pm 2,2 \times 104$ & $2,3 \times 105 \pm 4,6 \times 104$ & $3,2 \times 105 \pm 5,5 \times 104$ \\
Cocos gram-positivos & $7,7 \times 104 \pm 3,8 \times 104$ & $6,9 \times 105 \pm 1,4 \times 105$ & $9,9 \times 105 \pm 1,7 \times 105$ \\
Cocos gram-negativos & $2,6 \times 104 \pm 1,3 \times 104$ & $1,7 \times 105 \pm 4,5 \times 104$ & $1,9 \times 105 \pm 3,3 \times 104$ \\
Bacilos gram-negativos & $1,0 \times 104 \pm 5,1 \times 103$ & $4,5 \times 104 \pm 1,2 \times 104$ & $4,5 \times 104 \pm 7,9 \times 103$ \\
Microbiota total & $1,5 \times 105 \pm 7,7 \times 104$ & $1,1 \times 106 \pm 2,4 \times 105$ & $1,5 \times 106 \pm 2,6 \times 105$ \\
\hline \hline
\end{tabular}

cocos gram-positivos se recuperaron en mayor número en todas las muestras en relación con la microbiota restante.

Las diferencias en el recuento de la microbiota total, entre el grupo de estudiantes sanos y los sujetos con riesgo cariogénico y con actividad cariogénica, fueron estadísticamente significativas $(\mathrm{p}<0,05)$. (Figura 1$)$

En la Tabla 2, se indican las Desviaciones Estándar de cada morfotipo microbiano y de la microbiota total recuperada de saliva en los 3 grupos clínicos estudiados.

3) Recuento Actinomyces en saliva: En todos los grupos estudiados se aislaron Actinomyces en saliva. Los niveles en saliva de estos microorganismos entre los estudiantes sanos y aquellos con riesgo y actividad cariogénica mostraron diferencias estadísticamente significativas $(\mathrm{p}<0,05)$.

Cuando se comparó el número de estos microorganismos entre el grupo con riesgo y con actividad ca-

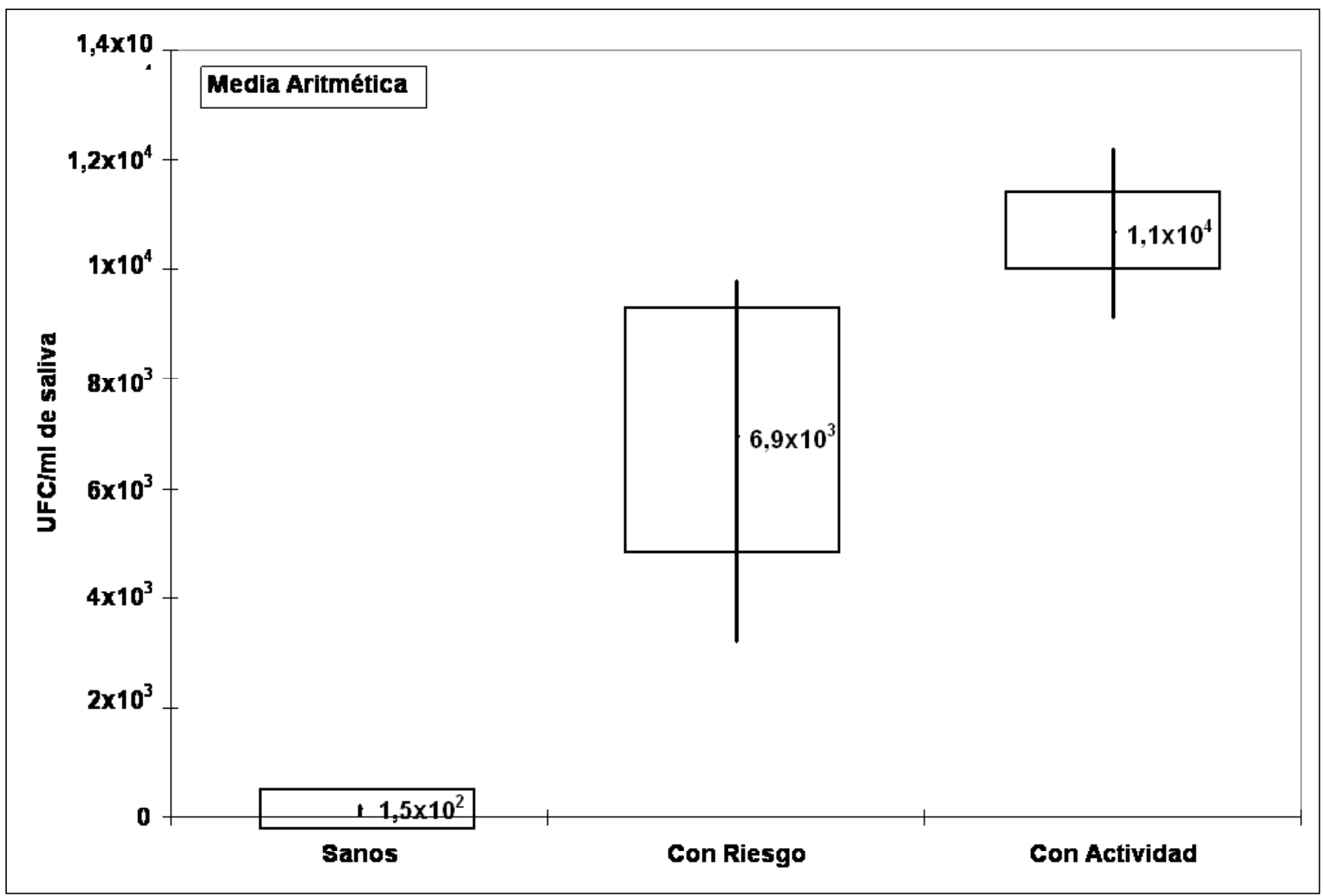

Figura 2: Recuento de Actinomyces en los 3 grupos estudiados 
Gutiérrez de Annan S, Benito de Cárdenas L. Recuento de actinomyces en saliva como predictores microbiológico de caries Revista de Odontologia da Universidade Cidade de São Paulo 2009 jan-abr; 21(1): 6-13

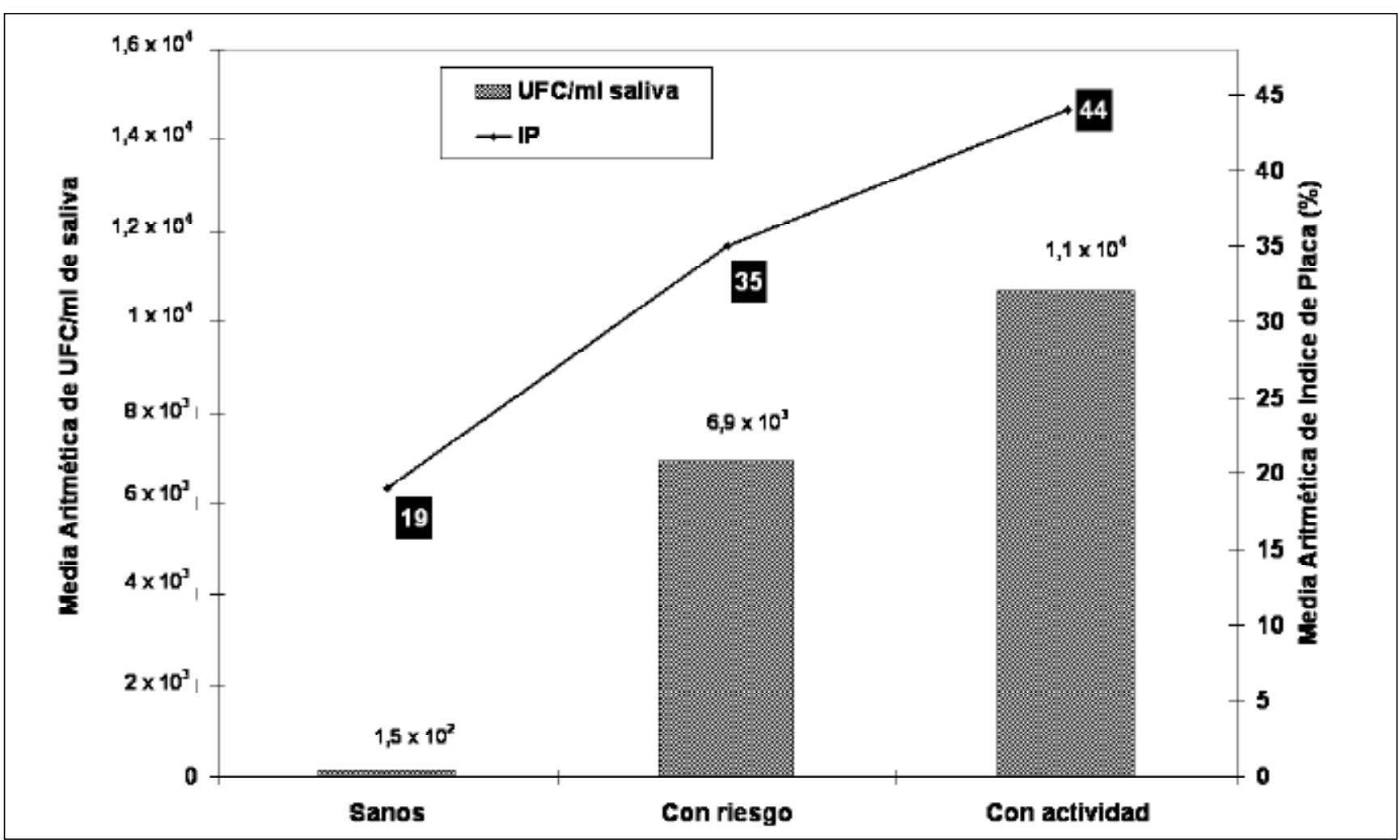

Figura 3: Relación entre el Índice de Placa O'Leary (IP) y el recuento de Actinomyces

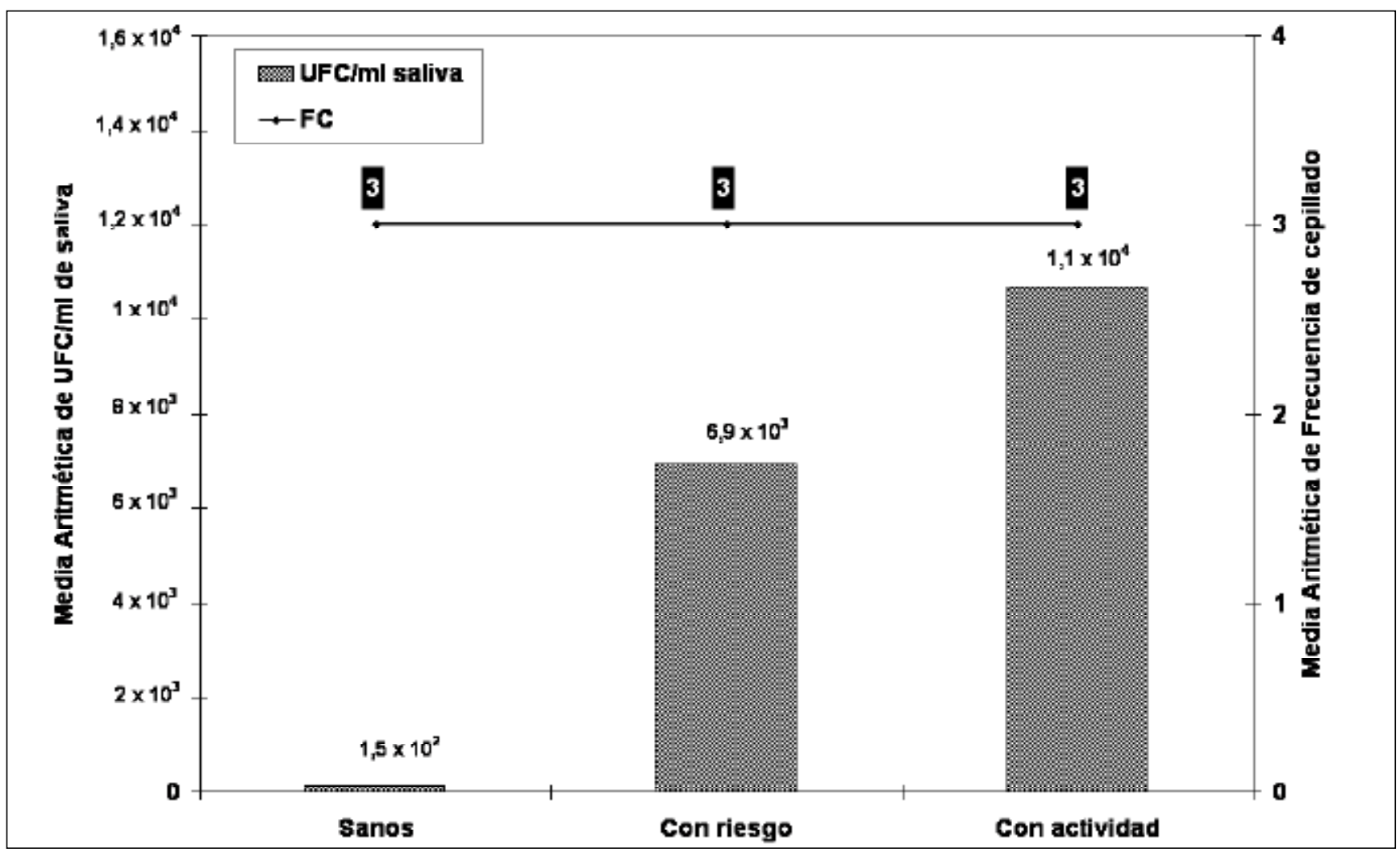

Figura 4: Relación entre la frecuencia de cepillado y el recuento de Actinomyces 
Gutiérrez de Annan S, Benito de Cárdenas L. Recuento de actinomyces en saliva como predictores microbiológico de caries Revista de Odontologia da Universidade Cidade de São Paulo 2009 jan-abr; 21(1): 6-13

riogénica también se encontró diferencias significativas $(\mathrm{p}<0,05)$. (Figura 2)

4) Relación entre el Índice O'Leary y Recuento de Actinomyces en saliva: Se encontró que hay una correlación directa entre el Índice de placa O'Leary y el recuento de Actinomyces. (Figura 3)

5) Relación entre Caries Amelodentinarias Abiertas y la Presencia de Actinomyces en saliva: Se encontró que el nivel de Actinomyces en saliva es mayor en los estudiantes que presentan caries amelodentinarias abiertas. El análisis estadístico de los datos indica una correlación directa entre estos dos parámetros. (Tabla 3)

6) Relación entre Frecuencia de Cepillado y la Presencia de Actinomyces en saliva: Se encontró que no hay asociación entre la frecuencia de cepillado (1 a 3 por día) y la cantidad de Actinomyces en saliva. (Figura 4)

7) Relación entre Momentos de Azúcar y la Presencia de Actinomyces en saliva: Cuando se relacionó los momentos de azúcar con los niveles de Actinomyces en saliva se encontró una correlación directa entre ambas variables. (Figura 5)
Tabla 3: Relación entre el estado de salud bucal, el número de Actinomyces en saliva y las cavidades amelodentinarias abiertas

\begin{tabular}{ccc}
\hline \hline $\begin{array}{c}\text { Estado de salud } \\
\text { bucal }\end{array}$ & UFC/ml saliva & $\begin{array}{c}\text { Cavidades } \\
\text { amelodentinarias } \\
\text { abiertas }\end{array}$ \\
\hline Sanos & $1,5 \times 102$ & $0 \pm 0$ \\
Con riesgo & $6,9 \times 103$ & $0 \pm 0$ \\
Con actividad & $1,1 \times 104$ & $3 \pm 1$ \\
\hline \hline
\end{tabular}

\section{DISCUSIÓN Y CONCLUSIONES}

El análisis de los factores primarios y secundarios que intervienen en la iniciación y progresión de la caries dental permite, actualmente, adoptar un criterio preventivo, en vez del criterio restaurativo al que tienden la mayoría de los profesionales.

Las investigaciones llevadas a cabo en los últimos años en relación con la cariología están dirigidas a reconsiderar las estrategias diagnósticas tradicionales de manera que se pueda reorientarlas hacia nuevas técnicas de diagnóstico de esta enfermedad y de esta manera poder combatir la caries desde sus primeros estadíos.

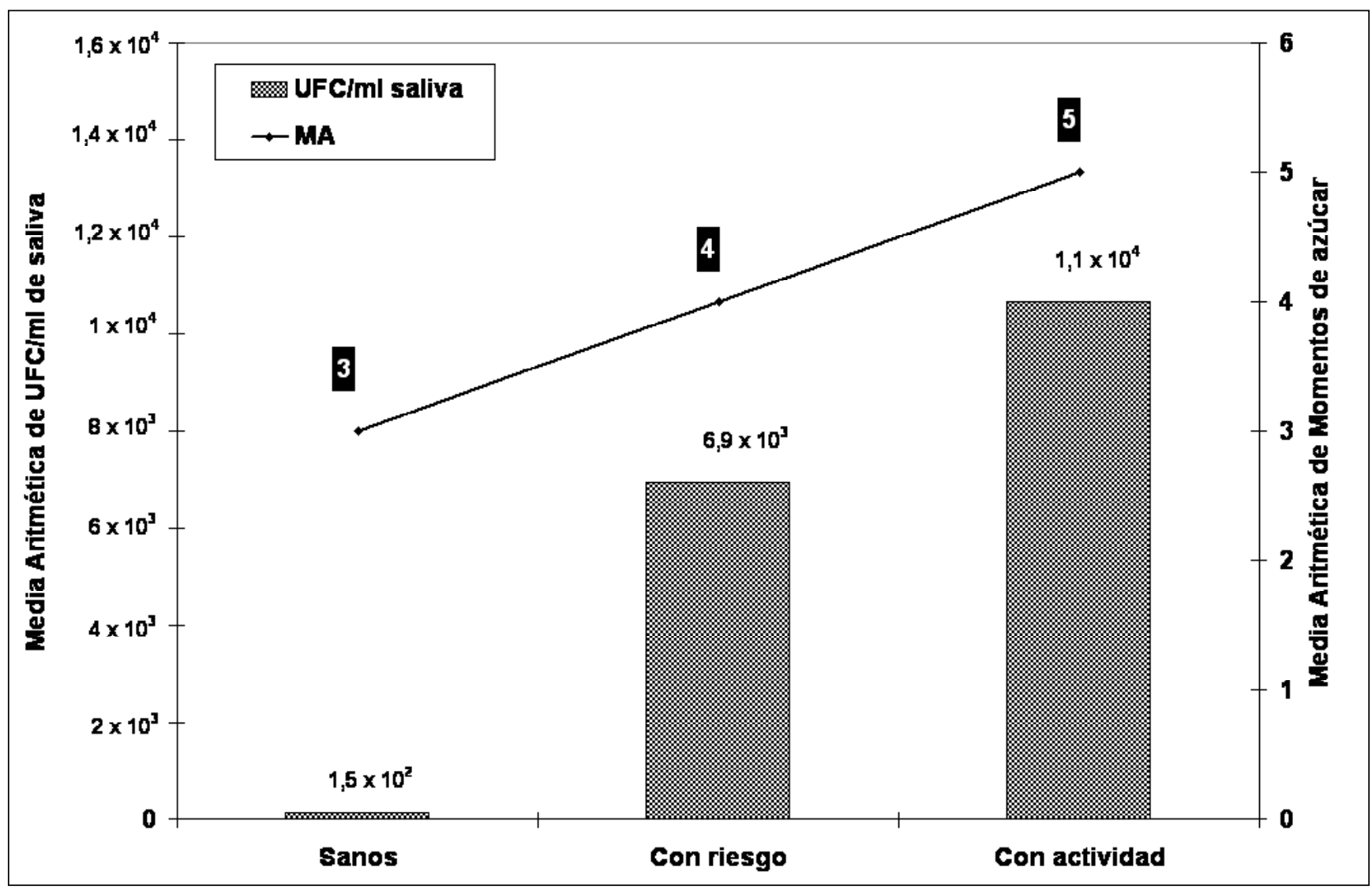

Figura 5: Relación entre momentos de azúcar y el recuento de Actinomyces 
Uno de los problemas principales es la cotidiana costumbre de diagnosticar caries una vez que ya se observa la destrucción de los tejidos duros del diente. El tratamiento impartido es la eliminación de la lesión, ya que si no se actúa rápidamente, ésta continuará hasta una patología pulpar con la consecutiva pérdida dentaria. $\mathrm{Si}$ bien esto es válido, hoy en día sabemos que disponemos de nuevas herramientas, mediante las cuales, la caries puede predecirse, prevenirse y aún detenerse en lesiones iniciales. (Bowden', 1997)

Hasta el presente se ha utilizado el recuento, en saliva, del grupo Streptococcus mutans y Lactobacillus como pruebas complementarias del diagnóstico clínico de la caries dental. Varios investigadores están actualmente abocados al estudio del rol que cumplen las especies del género Actinomyces como patógenos oportunistas y se los está correlacionando con caries dental. Estos microorganismos jugarían un papel importante en la iniciación de esta enfermedad. (Sarkonen $\left.e t a l^{1}, 2000\right)$ (Becker $e t$ $a \bar{P}, 2002)$

De acuerdo a los resultados obtenidos en esta muestra se puede concluir que:

El recuento de la microbiota total entre el grupo sano y con riesgo cariogénico y entre los estudiantes sanos y con actividad cariogénica fue estadísticamente significativa.

Hubo diferencias estadísticamente significativas en el recuento de Actinomyces entre los estudiantes sanos y aquellos que clínicamente fueron clasificados con riesgo cariogénico o que presentaron actividad cariogénica. En este último caso el nivel de microorganismos fue significativamente alto, lo que indica que están colonizando los tejidos duros de la boca, especialmente en aquellos sitios en donde ya está presente la cavidad abierta.

Se encontró una correlación directa estadísticamente significativa entre el recuento de Actinomyces y el Índice de Placa O'Leary, el número de Caries Amelodentinarias Abiertas y los momentos de azúcar.

No se encontró asociación entre el número de Actinomyces con la frecuencia de cepillado.

El análisis del presente estudio, en donde se encontró diferencias en el número de Actinomyces en saliva entre el grupo de estudiantes sanos y aquellos que tenían riesgo cariogénico, conduce a proponer la utilización del recuento de estos microorganismos como predictores de riesgo de caries conjuntamente con el recuento del grupo Streptococcus mutans, Lactobacillus y Candida

Es importante continuar con futuras investigaciones sobre la relación de estos microorganismos con caries dental en otros grupos etarios y con un número mayor de individuos.

\section{AGRADECIMIENTOS}

Este trabajo fue subsidiado por el Consejo de Investigación de la Universidad Nacional de Tucumán.

\section{REFERENCIAS}

1. Almagro-Nievas D, Benítez-Hita JA, García-Aragón MA, López-Lorca MT. Incremento del índice de dientes permanentes cariados, perdidos por caries y obturados, entre escolares de loja, España. Salud Pública de Méx 2001 May-Jun; 43(3): 192-8.

2. Axelsson P, Lindhe J, Nystrom B. On the preventive of caries and periodontal disease: results of a 15year longitudinal study in adults. J. Clin. Periodontol 1991 Mar; 18(3): 182-9

3. Baca García P, Liébana Ureña J. Microbiología de la caries dental. En: Liébana Ureña J. Microbiología Oral. Madrid. España: Interamericana; 1995 cap. 32 p 454.
4. Balda Zavarce R, González Blanco O, Solórzano Peláez L. Evaluación del riesgo de la caries dental como un proceso infeccioso. Propuesta de un modelo para historia clínica. Acta Odontol Venez 1999; 37(3): 106-11

5. Becker MR, Paster BJ, Leys EJ, Moeschberger ML, Kenyon SG, Galvin JL, et al. Molecular analysis of bacterial species associated with childhood caries. J. Clin Microbiol 2002 Mar; 40(3): 1001-9

6. Beighton D. The complex microflora of high-risk individuals and groups and its role in the caries process. Community Dent Oral Epidemiol 2005 Aug; 33(4): 248-55 
7. Bellini HT, Arneberg P, Von Der Fehr FR. Oral hygiene and caries. A review. Acta Odontol Scand 1981; 39(5): 257-65

8. Benito de Cárdenas IL, Gutierrez de Annan ES, Testa de Nadal MM. Efecto del tratamiento preventivo sobre la microflora salival. recuento de estreptococos. Rev Asoc Odontol Argent 1994Abr-Jun; 82(2): 126-31

9. Bowden GH. Does assessment of microbial composition of plaque/saliva allow for diagnosis of disease activity of individuals. Community Dent Oral Epidemiol 1997 Fev; 25(1): 76-81

10. Cortelli SC, Chaves MGAM, Faria IS, Landucci LF, Oliveira LD, Scherma AP, et al. Avaliação da condição bucal e do risco de cárie de alunos ingressantes em curso de odontología. Rev Fac Odontol Sao José dos Campos 2002 jan-abr; 5(1): 35-42

11. Elderton RJ, Mjör IA. Changing scene in cariology and operative dentistry. Int Dent J 1992 Jun; 42(3): 165-9

12. Fejerskov O. Changing paradigms in concepts on dental caries: consequences for oral health care. $\mathrm{Ca}$ ries Res 2004 May-Jun; 38(3): 182-91

13. Fejerskov O. Concepts of dental caries and their consequences for understanding the disease. Community Dent Oral Epidemiol 1997Feb; 25(1): 5-12

14. Garrity G.M, Winters M, Searles DB. Taxonomic outline of the procaryotic genera. In: Bergey's Manual of systematic bacteriology. 2nd ed. New York: Springer 2001; p. 20

15. Gutiérrez Acero D, Alós Cortés L, García Gómez F, González Sanz A. Microbiología de la caries radicular en el paciente mayor. Av Odontoestomatol 2006 Mar-Apr; 22(2): 125-130

16. Hausen H. Caries prediction state of the art. Community Dent Oral Epidemiol 1997 Feb; 25(1): 87-96
17. Johnson JL, Moore LV, Kaneko B, Moore WE. Actinomyces georgiae sp.nov., Actinomyces gerencseriae sp.nov., designation of two genospecies of Actinomyces naeslundii and inclusion of $A$. naeslundii serotypes II and III and Actinomyces viscosus serotype II in $A$. naeslundii genospecies 2. Int J Syst Bacteriol 1990 Jul; 40(3): 273-86

18. Kandler O, Weiss N. Regular, nonsporing gram-positive rods. In: Willian ST, Sharp E, Holt JG. (Editors). Bergey's manual of systematic bacteriology. 9 nd ed., Baltimore: Williams and Wilkins, 1986 v.2 p.12081417

19. Ismail AI. Clinical diagnosis of precavitate carious lesions. Community Dent Oral Epidemiol 1997Fev; 25(1): 13-23

20. Loyo Molina K, Balda Zavarce R, González Blanco O, Solórzano Peláez AL, González AM. Actividad cariogénica y su relación con el flujo salival y la capacidad amortiguadora de la saliva. Acta Odontol Venez 1999; 37(3): 10-7

21. Sarkonen N, Könönen E, Summanen P, Kanervo A, Takala A, Jousimies-Somer H. Oral colonization with Actinomyces species in in-fants by two years of age. J Dent Res 2000 Mar; 79(3): 864-7

22. Tenovuo J. Salivary parameters of relevance for assessing caries activity in individuals and populations. Community Dent Oral Epidemiol 1997Fev; 25(1): 82-6

23. Thenisch NL, Bachmann LM, Imfeld T, Leisebach Minder T, Steurer J. Are mutans streptococci detected in preschool children a reliable predictive factor for dental caries risk? A systematic review. Caries Res 2006. 40(5): 366-74

24. Van Houte J. Microbiological predictors of caries risk. Adv Dent Res. 1993 Aug; 7(2): 87-96

25. van Palenstein Helderman WH, Soe W, van't Hof MA. Risk factors of early childhood caries in a Southeast Asian population. J Dent Res 2006 Jan; 85(1): 85-8.

Recebido em: 17/06/08 Aceito em: 16/10/08 